\title{
ene
}

\section{REPERCUSIONES DE PADECER UNA ÚLCERA VENOSA}

\section{REPERCUSSIONS OF SUfFERING A VenOUS ULCER}

\author{
Cristo Manuel Marrero González \\ Servicio Canario de la salud. Área de Salud de Tenerife (España). Doctor por la \\ Universidad de La Laguna (España)
}

\footnotetext{
Marrero González, C. (2020). Repercusiones de padecer una úlcera venosa. Revista Ene De Enfermería. 14(2) 


\section{Resumen}

Las úlceras venosas suponen un problema de salud para el paciente muy importante afectando además a una parte de las personas mayores de 65 años. Padecer de una úlcera venosa implica complicaciones como dolor continuado, soportar mal olor desprendido desde la propia herida, tener limitaciones funcionales para poder deambular y posibles problemas de adherencia terapéutica. Este ensayo tiene como objetivo mostrar las repercusiones que a nivel biopsicosocial tienen para una persona padecer una herida de estas características. Se muestra que no solo está afectada una pierna, sino una persona en su conjunto ya que estas lesiones terminando afectando a la persona a nivel físico, funcional, emocional, social, laboral e incluso a nivel de realización personal. Se propone una atención por parte de enfermería integral y que además se desarrolle la figura de la enfermera consultora de heridas con una vocación de atención holista y no funcionalista.

Palabras clave: úlcera venosa, fenomenología, investigación cualitativa, atención integral.

\section{Abstract}

Venous ulcers pose an important health problem, especially for people over 65 years of age. Having a venous ulcer involves complications such as continued pain, enduring a bad smell from the wound itself, functional limitations to be able to wander and possible problems of therapeutic adherence. The purpose of this paper is to show the repercussions that a person with this type of injury has on a biopsychosocial level. It shows that not only one leg is affected, but a person as a whole as these injuries end up affecting the person physically, functionally, emotionally, socially, work and even at the level of personal fulfillment. It is proposed a comprehensive nursing care and also develop the figure of the wounds consultant nurse with a holistic vocation and non-functionalist care.

Key Words: venous ulcer, phenomenology, cualitative investigation, comprehensive care. 


\section{RESEÑA INTRODUCTORIA}

Las úlceras venosas son consideradas un problema de salud de gran envergadura ya que suponen un problema importante para quien las padece a nivel holístico en cuanto a dolor, funcionalidad física, movilidad, depresión y aislamiento afectando obviamente a la calidad de vida del paciente. Las úlceras venosas suponen un gasto importante para el sistema de salud por sus cronicidades y sus recidivas. Las úlceras venosas representan el $75-80 \%$ de las lesiones aproximadamente, teniendo en España una prevalencia que se sitúa entre 0,8 y $0,5 \%$ afectando en mayor proporción a mujeres que a hombres y al grupo de edad de mayores de 65 años (Blanco et al, 2016). Además, el envejecimiento se caracteriza a día de hoy como un problema mundial y en relación a este fenómeno están las úlceras venosas en la tercera edad (Sameiro Neves, 2017).

Como destaca Madoux (2012), aspectos como el dolor, el exceso de exudado del lecho ulceral, o el olor que desprende dicho exudado, son circunstancias que afectan de forma notable al bienestar psicológico y emocional de la persona que sufre de una úlcera venosa. De igual modo se ve afectada la vida social del paciente, ya que se ve limitado en su vida diaria.
Con el presente ensayo, pretendo ahondar en las vivencias que tienen los pacientes que padecen una úlcera venosa de forma integral y las repercusiones que tienen este tipo de heridas en sus calidades de vida. El objetivo que me planteo con este manuscrito es el de tratar de describir de forma concisa dichas vivencias y de alguna manera tratar de defender una atención holista del paciente, no funcionalista. El ser humano se vertebra como un todo. La úlcera crónica no es un aspecto que solo afecta a la pierna del paciente. Afecta a la persona en su funcionalidad, en su día a día, en su vida social, en su vida íntima e incluso en su realización como persona ya que seguramente muchos de sus proyectos vitales se habrán aparcado por completo. La herida ya forma parte de su vida por completo, y de todas sus esferas vitales. Cualquier aspecto de su vida ya está construido a causa de esa úlcera que se encuentra en esa pierna. Mi justificación para llevar a cabo este ensayo se sustenta también en que en mi formación académica y científica sólo se ha centrado en la esfera somática. También debo resaltar que en España y en la cultura de la enfermería española se pasa por alto la esfera más humana en el tratamiento de una herida crónica existiendo escasa literatura española. 


\section{VIVENCIAS DE LOS PACIENTES} CON ÚLCERAS

La presencia de una herida crónica en una persona repercute de forma importante en su vida social. Esto crea un sufrimiento en el paciente, ya que se deteriora su esfera social y sus relaciones sociales. La herida hace presentar limitaciones en su sociabilidad: la persona con una úlcera en la pierna relata sentirse angustiada en lo social, llegando incluso a marginarse (Aguiar et al, 2016). Las úlceras venosas producen dolor en la persona que las padece. Las úlceras venosas además se caracterizan por presentar contenidos de exudados altos, lo que conlleva a que se desprenda mal olor y unido al dolor afecta de forma negativa en el bienestar psicológico y emocional del paciente y en la ya mencionada interacción social (Maddox, 2012).

Está evidenciado que una úlcera venosa afecta de forma notable en la calidad de vida del paciente y de que interfiere en su vida diaria (Dos Santos et al, 2015).

Gonring Xavier (2016), habla de que padecer una úlcera sobrepasa los límites de una dimensión meramente biofisiológica. La presencia de esta lesión en la piel es sentida en múltiples dominios de la existencia humana. El cuidado y tratamiento de estas heridas puede extenderse durante largos períodos de tiempo, incluso en algunos casos duran- te décadas. Una úlcera modifica todos los hábitos cotidianos de la persona que la padece: su patrón de sueño y descanso, su higiene diaria y su vida social está mucho más limitada. Se evita visitar playas, piscinas o parques o realizar deporte. Todas estas circunstancias llevan a la persona al aislamiento social, la tristeza y la depresión.

Las mujeres que sufren de una herida crónica encuentran dificultades a la hora de realizar los autocuidados relacionados con la higiene, el vestido y el encontrar un calzado adecuado y adaptado para el cuidado de la herida. Muchas de las dificultades que expresan las mujeres con úlceras vasculares están relacionadas con su adherencia al tratamiento de la terapia compresiva y al tratamiento tópico local que se aplica en los lechos ulcerales. La intensidad del dolor de una úlcera vascular influye en el patrón de sueño de la paciente y también en su alimentación. Debido a que la mayoría de mujeres son amas de casa o llevan el cuidado de la familia en el hogar, no pueden llevar a cabo adecuadamente sus autocuidados con una herida no pudiendo guardar el suficiente reposo debido a las tareas del hogar. Las mujeres no suelen recibir suficiente apoyo familiar para el cuidado del hogar y hasta incluso han de cuidar a sus propios nietos (Da Silva et al, 2016). El dolor es una 
de las vivencias más importantes a lo largo de la cronicidad que se palia con analgésicos, reposo, elevación del miembro inferior afectado y masaje para mejorar el retorno venoso (Gonning Xavier, 2016).

Las mujeres expresan sentimientos de felicidad o libertad cuando hablan de una posible cicatrización, ya que eso supondría reanudar una vida social aparcada y considerarse a sí mismas que gozan de mejor salud (Da Silva et al, 2016). En el cuidado de la mujer que padece de una úlcera, se aprecia en la misma baja autoestima debido al deterioro de su imagen y el carácter recidivante de estas lesiones hace desmotivar a la mujer (Da Silva, 2012).

En general, las personas que sufren de una úlcera venosa crónica manifiestan sentimientos de esperanza ante el hecho de que se pueda cicatrizar la lesión. Que se epitelice la herida, significa poder recuperar la vida social perdida, afectada por el olor, el dolor, la apariencia de la pierna, por la restricción en el uso de ciertos calzados o vestimentas además de las limitaciones a nivel laboral y de ocio debido al cuidado de los vendajes compresivos. De hecho, la aplicación de ciertos métodos de compresión venosa produce dolor y malestar en el paciente (Da Silva et al, 2017).
Do Rego Sousa (2009), coincide en destacar que el hecho de que una úlcera venosa afecte a una persona a realizar las actividades más básicas y banales de la vida diaria hace que el paciente se sienta prisionero, sin libertad individual por dicha restricción de vida que padece. Incluso el paciente manifiesta sentimientos de injusticia a nivel laboral o profesional porque no puede permanecer tanto tiempo de pie como el resto de sus compañeros/as.

Ouro et al (2017), nos habla de que los hombres que padecen de una herida crónica expresan sentimientos negativos como tristeza, melancolía, impotencia, aislamiento social y afectación en la autoestima ya que perciben una autoimagen deteriorada. Los hombres expresan problemas a nivel sexual tras la aparición de una úlcera en la piel a causa del dolor, del olor desprendido por la herida, por el temor a que haya accidentes con los vendajes o el temor de que la pareja perciba mal olor. Otros factores que refieren los autores que afectan a la sexualidad del hombre están relacionados con la fe, las creencias religiosas, la cultura, los factores económicos, sociales, de género, geográficos y educativos. Todos estos problemas a nivel biopsicosocial incluyendo la sexualidad o la vida social del hombre, aparecen en el hombre por el constructo de 
género masculino que se da en las sociedades occidentales.

Da Silva et al (2013), coinciden con los anteriores autores en que para los hombres padecer una ulcera en la piel supone un problema para su autoimagen corporal y para la percepción que tienen de sí mismos hacia los demás. Cabe destacar que debido al sufrimiento del dolor o del olor del exceso de exudado de la herida, hace que sus vidas a nivel social se vean truncadas con respecto al trabajo o al ocio. Padecer de una úlcera supone largos períodos de incapacidad laboral y dificultades para deambular. A consecuencia de esto, los pacientes pueden verse abocados a jubilarse de forma prematura provocando así cambios económicos, emocionales y sociales (Da Silva et al, 2015). Cabe señalar además que las personas con bajos ingresos económicos y socialmente vulnerables que padecen una úlcera venosa, tienen peor calidad de vida (De Oliveira et al, 2016).

\section{PAPEL DE ENFERMERÍA}

La presencia de una herida crónica repercute en el plano social de la persona como hemos señalado. Tratar a una persona mayor, por ejemplo, con una herida crónica requiere de las enfermeras no sólo competencias científicas, sino también de un cuidado sensible y guiado. Para comprender la herida no se puede hacer desde una visión aislada o fragmentada. Se debe mirar más allá de la lesión y comprender a la persona como un ser que tiene sentimientos y necesidades a nivel integral. En la formación de enfermería en las universidades se debe invertir en enseñar a las futuras enfermeras desde esta perspectiva, enfoque o visión en el cuidado holístico del ser. El cuidado de la persona mayor con una herida significa un desafío para enfermera. Conocer las repercusiones sociales en la persona anciana ayudará a las profesionales a prestar una atención integral, interdisciplinaria y capacidad para enfrentar las adversidades y el estigma social de convivir con una herida (Aguiar et al, 2016). Al comprender al ser humano desde una multidimensión humana, se considera a la persona algo mucho más que la suma de sus partes (Gonning Xavier, 2016). La enfermera al ser la profesional más cercana al paciente ha de garantizar una asistencia humanizada a fin de conocer a la persona en su totalidad llevando un cuidado personalizado para lograr una intervención que facilite lo más pronto posible la cicatrización y prevenir las recidivas (Sameiro Neves, 2017).

Es competencia de enfermería realizar una labor de educación para la salud en pro de prevenir complicaciones 
e identificar los factores de riesgo al acoger al paciente, escucharlo activamente y teniendo mucho diálogo con el mismo esclareciendo dudas de manera continua. En esta labor de educación para la salud también se ha de hacer partícipe a los familiares y/o cuidadores/ as del paciente (Gonning Xavier, 2016).

Se debe hacer hincapié por tanto en una atención holística, necesaria para el tratamiento del paciente con úlcera venosa y para lograr mejorar los resultados del paciente y por ende su calidad de vida (Maddox, 2012). Por ejemplo, cuidar de un anciano que sufre de una herida trasciende más allá de un simple vendaje. Es necesario adentrarse en la subjetividad de la persona y comprender sus angustias, percepciones, miedos, tristezas y expectativas (Aguiar et al, 2016).

El profesional de enfermería especializado o competente en heridas crónicas no solamente ha de tratar la úlcera en sí sino también tratar al paciente de forma integral valorando sus creencias, su situación económica, su situación emocional, su estado nutricional y sus funciones sistémicas alteradas. Todos estos factores interfieren directamente en el proceso de cicatrización. Se trata de concebir a la persona como un ser dentro de una historia de vida, dentro de una cultura, comprendiendo sus ne- cesidades y expectativas (Da Silvera, 2016). Así pues, Gonning Xavier (2016), insiste en que la formación académica universitaria en heridas y la formación posgrado se ha de llevar a cabo desde un enfoque holístico y desde una práctica basada en la evidencia, con investigaciones actualizadas en el control del dolor. Silva y Pazos (2005), insisten en que el tratamiento del dolor implica un enfoque humano amplio, con el que el paciente se pueda rehabilitar globalmente tanto en su parte física, como social. Proporcionándose así una mejor calidad de vida.

El tratamiento de una herida crónica y la consecución de los resultados de cicatrización resulta ser un objetivo complejo tanto para el paciente que la sufre como para la enfermera (Gonning Xavier, 2016). Da Silva, et al (2017), argumentan que las enfermeras pueden sentirse frustradas o desmotivadas al tratar la herida por las recidivas que presentan las úlceras como el escaso trabajo interdisciplinar. Las enfermeras también presentan frustración por la falta de adherencia al tratamiento por parte del paciente, dadas las dificultades que presentan éstos y que se han mencionado anteriormente. En el tratamiento de la úlcera venosa, es necesario desarrollar la implicación e involucración del paciente y desarrollar su propia resiliencia. 
Pero además se han de involucrar los demás profesionales como médicos, estomaterapeutas, gestores sanitarios y las instituciones sanitarias con el fin de mejorar el trabajo multidisciplinar. Las orientaciones profesionales hacia el paciente deben ser orientadas hacia un autocuidado efectivo, conociendo sus realidades y ayudándole a sobrellevar las dificultades en torno a su padecer (Da Silva, 2016).

Por lo tanto, como indica Da Silvera (2016), se hace necesaria la elaboración de protocolos institucionales para estandarizar los cuidados. Se requiere que en cada zona o en ciertas áreas poblacionales, existan equipos multidisciplinares competentes que abarquen el cuidado integral de por ejemplo los ancianos que sufren de una herida crónica atendiendo no solo el cuidado de la lesión. Dos Santos et al (2015), muestran que la planificación de cuidados garantiza una atención de calidad y genera mejores resultados de curación, de funcionalidad y mejora la percepción del dolor contribuyendo al bienestar y calidad de vida de las personas con úlceras crónicas. Este tipo de pacientes requiere de seguimiento periódico por parte del equipo multidisciplinar. Los equipos multidisciplinares, y en especial las enfermeras, al promover una atención integral han de tratar que las barreras de salud socio- demográficas afecten lo menos posible en la recuperación del paciente que presenta herida crónica. Como se ha venido insistiendo, la escucha y orientación al paciente, familiares y cuidadores/as es importante (De Oliveira, et al 2016).

\section{CONSIDERACIONES FINALES POR PARTE DEL AUTOR}

He de reconocer que me ha congratulado y mucho el hecho de que exista producción investigadora de corte fenomenológico en el que muchos/as autores/as se haya preocupado por esa idea que en la reseña introductoria planteaba: concebir al ser humano no solo como algo físico sin sentido. La herida no está aislada, está presente en un cuerpo que tiene "alma". La herida no solo se limita a los bordes perilesionales de lecho ulceral. Esa úlcera tiene una causa de producción, ese ser humano que la sufre tiene muchos otros padeceres que se han relacionado con esa herida. Las personas son seres sociales en relación y como se ha ido desarrollando en este humilde manuscrito, la herida va a suponer cambios en la persona de forma holista. Ahora la vida se construye y se limita dicha vida a la herida y al tratamiento.

Me ha sorprendido la cantidad de producción investigadora de carácter fenomenológico que he consultado proce- 
dente de Brasil y tan escasa o nula procedente de mi país, España. Sin embargo, como expone Da Silva (2012), pese a que existen grandes avances científicos y tecnológicos que se han desarrollado para el tratamiento de heridas, existe escasa literatura en cuanto a investigaciones de metodología cualitativa. El paradigma español de enfermería está muy centrado en la parte somática casi obviando el resto de las áreas del ser humano y que influyen en la curación de una herida desde el estado emocional al estado socioeconómico.

No obstante, en España se está intentando desarrollar las unidades clínicas multidisciplinares y la figura de la enfermera consultora en heridas que ya existe en muchos países del entorno de España (Soldevilla-Agueda et al, 2016). Tal vez con el desarrollo de esta figura profesional especializada, pero, y espero, que no olvide que el ser humano es un ser holista y no funcionalista, se mejore la atención de los pacientes que sufren de heridas crónicas que como hemos visto no se trata solo de una pierna sino de un ser humano como tal. El desarrollo de investigaciones cualitativas como etnografías o estudios fenomenológicos, nos puede ayudar a dar una visión más humana y más completa del ser humano y a ayudar a dar a la enfer- mería ese espíritu en la humanización de cuidados. 


\section{BIBLIOGRAFÍA}

Aguiar ACSA., Sadigursky D., Martins LA., Menezes TMO., Santos ALS. \& Reis LA. (2016). Repercussões Sociais Vivenciadas Pela Pessoa Idosa Com Úlcera Venosa.

Rev. Gaúcha Enferm.,37(3), e55302. Doi: 10.1590/1983- 1447.2016.03.55302.

Blanco-Blanco, J., Ballesté-Torralba, J. \& BerenguerPérez, M. (2016). Úlceras Venosas. En: García-Fernández, FP., Soldevilla -Agreda, JJ., Torra Bou, JE. Atención Integral de las Heridas Crónicas (Volumen II) $-2^{\text {a }}$ Edición-. Logroño: GNEAUPP-FSJJ.

De Oliveira Torres, S., Araújo, R., Oliveira, R., Pergola-Marconato, A., Mansano-Schlosser, T., Ecco, L., da Silva, D., Torres, G., \& Chaves Maia, E. (2016). Care and Quality of Life Evaluation of People with Venous Ulcers in Primary Health Care. International Archives Of Medicine, 9(216), 1-10. Doi: $10.3823 / 2087$.

Da Silva, D. C., Denardin Budó, M. D. L., Schimith, M. D., Ecco, L., Fernandes Costa, I. K., \& de Vasconcelos Torres, G. (2015). Experiências construídas no processo de viver com a úlcera venosa. Cogitare Enfermagem, 20(1), 13-19.

Da Silva, M. (2012). Experiências e Expectativas de Enfermeiras em Relaçao ao Cuidado à Mulher com Úlcera Venosa Crônica (Dissertação). Escola de Enfermagen. Universidade de São Paulo. Brasil.

Da Silva, M., Pinto de Jesus, M., Barbosa Merighi, M. A., Moura de Oliveira, D., Biscotto, P. R., \& Silva, G. P. S. (2013). The Daily Life of Men who Lives with Chronic Venous Ulcer: Phenomenological Study. Revista gaucha de enfermagem, 34(3), 95-101. Doi: 10.1590/S1983-14472013000300012

Da Silva, M., Pinto de Jesus, M., Barbosa Merighi, M., \& Moura de Oliveira, D. (2014). Limites e possibilidades vivenciados por enfermeiras no tratamento de mulheres com úlcera venosa crônica. Revista da Escola de Enfermagem da USP, 48 (1), 54-59. Doi: 10.1590/S0080-623420140000600008

Da Silva, M., Pinto de Jesus, M., Barbosa Merighi, M., \& Moura de Oliveira, D. (2016). A Experiência de Autocuidado de Mulheres que Convivem com Úlcera Venosa Crônica. Revista Estima, 14(2), 61-67. Doi: 10.5327/Z1806-3144201600020003.

Da Silva, M., Pinto de Jesus, M., Moura de Oliveira, D., \& Barbosa Merighi, M. A. (2017). Bota de Unna: Vivência do Cuidado por Pessoas com Úlcera Varicosa. Revista Brasileira de Enfermagem, 70(2), 366373. Doi:10.1590/0034-7167-2016-0219.

Da Silvera, L. (2016). Cuidado às Pessoas Portadoras de Lesões crônicas de Pele: Percepções das Enfermeiras da Atenção Primária à Saúde (Trabalho de Conclusão do Curso de Especialização em Cuidado Integral com a Pele no Âmbito da Atenção Básica). Escola de Enfermagen. Departamento de As- sistencia e Orientação Profissional. Universidade Federal Do Rio Grande Do Sul. Brasil.

Do Rego Sousa, F.A.M. (2009). O "Corpo" que não Cura. Vivências das Pessoas com Úlcera Venosa Crónica de Perna (Dissertaçao de candidatura ao grau de Mestre). Instituto de Ciéncias Biomédicas Abel Salazar. Universidade do Porto. Portugal.

Dos Santos, L. D. S. F., Leite Funchal Camacho, A. C., Renaud Baptista de Oliveira, B. G., Nogueira, G. D. A., \& Joaquim, F. L. (2015). Influence of Venous Ulcer in Patients' Quality of Life: an Integrative Review. Journal of Nursing UFPE on line, 9(3), 7710-7722. Doi:10.5205/reuol.7049-61452-1-ED. 0903 supl201526.

Gonring Xavier, F. (2016). Significado de Demandas de Cuidados de Pessoas que Vivenciam Úlceras Crônicas de Membros Inferiores: Contribuições para Enfermagem (Tese de doutorado). Escola de Enfermagen Anna Nery. Universidade Federal Do Rio de Janeiro. Brasil.

Lopes Joaquim, F. (2017). Úlcera Metafísica: um Constructo Teórico a Partir da Percepção Existencial de Pessoas com Úlceras Venosas Crônicas (Tese do doutorado). Escola de Enfermagem Aurora Afonso Acosta. Universidade Federal Fluminense. Brasil.

Maddox, D. (2012). Effects of Venous Leg Ulceration on Patient's Quality of Life. Nursing Standard, 26, (38), 42-49.

Ouro, F. L., Sousa, A. R., Moreira, W. C., dos Santos, W. N., Santana Carvalho, E. S., \& Pereira, Á. (2017). Impactos à sexualidade de homens que convivem com feridas crônicas: revisão integrativa. Revista de enfermagem UFPE, 11(2), 675-690. Doi: 10.5205/ reuol.10263-91568-1-RV.1102201723

Sameiro Neves, M. (2017). Importância da prestação dos cuidados de enfermagem aos idosos portadores de úlcera venosa: o processo de cicatrização (Trabalho de conclusão de curso de enfermagem). Escola Superior de Sáude. Universidade do Mindelo. Cabo Verde.

Silva, L. D. D., \& Pazos, A. L. (2005). A influência da dor na Qualidade de Vida do Paciente com Lesão Crônica de Pele. Rev. Enferm. UERJ, 13(3), 375-381.

Soldevilla-Agueda J.J, López- Casanova, P., García Fernández, F.P. (2016). Enfermeras Consultoras en Heridas Crónicas. En: García-Fernández, F.P., Soldevilla-Agreda, J.J., Torra Bou, J.E. Atención Integral de las Heridas Crónicas (Volumen II). - $2^{\mathrm{a}}$ edición-. Logroño: GNEAUPP-FSJJ. 